\title{
REVIEW
}

\section{Bacillus species of medical and veterinary importance}

\author{
N. A. LOGAN
}

\section{Department of Biological Sciences, Glasgow College of Technology, Cowcaddens Road, Glasgow G4 OBA}

In most current textbooks of medical microbiology, the chapter on Bacillus may well concentrate on Bacillus anthracis and give some information on $B$. cereus, now well established as a food-poisoning organism. A few recent texts may even indicate that $B$. cereus can be an opportunist pathogen but more usually this and other species of Bacillus will be dismissed as common contaminating organisms. They are, of course, important as contaminants; it would be surprising if it were otherwise, given their high numbers and ubiquity in natural, domestic and hospital environments (Finch et al., 1978) and their production of resistant endospores. Indeed, one study has shown that Bacillus spp. are the commonest bacterial contaminants of addicts' heroin and injection paraphernalia (Tuazon et al., 1974) and, as well as the sporadic appearance of Bacillus strains in routine diagnostic cultures, there have been reports of "pseudo-outbreaks" connected with contaminated clinical and laboratory equipment (Gurevitch et al., 1984; Goldstein and Abrutyn, 1985).

Bacillus species, however, have been known as opportunist pathogens since the late nineteenth century and reports of cases have appeared at the rate of about six a year for the past decade. Most of these reports commented on the danger of dismissing as mere contaminants Bacillus isolates recovered from specimens in the diagnostic laboratory and stressed how important it is to interpret their isolation in the light of other species cultured and the clinical context. There is little doubt, however, that many significant isolates have been, and will continue to be, discarded.

The increasing frequency with which opportunist infections are encountered is in part due to host predisposition by suppressed or compromised immunity, metabolic disorder, and malignant disease, or to exposure by surgical and clinical procedures, accidental trauma and drug abuse; it is also associated with advances in bacteriological technique and interpretation. Changes in the virulences

Received 18 Sep. 1987; accepted 30 Sep. 1987. of the microorganisms concerned seem unlikely. Indeed, were it not for the low invasiveness and virulence of most Bacillus species, the problem might be overwhelming.

The earlier literature on opportunistic infections by species of Bacillus has been reviewed by Norris et al. (1981) and reports before 1950 will not be considered in the present review.

\section{Anthrax}

Anthrax is a disease of great antiquity and historical interest; it may have been responsible for two of the biblical plagues of Egypt in $1491 \mathrm{BC}$ and its symptoms in animals were clearly described in $29 \mathrm{BC}$ by Virgil in the Georgics. As at least one cause of "Black Bane" and "Murrain" in Saxon and Medieval times, it caused heavy losses of cattle and sheep in Britain and Europe and it has been suggested that it contributed to the Black Death that swept Europe in the mid-fourteenth century (Twigg, 1984). Anthrax continued to be a problem in the nineteenth century and was responsible for mortalities of $25-50 \%$ in some European sheep flocks and for bringing French husbandry to a standstill. That situation encouraged the major research effort that led first in 1863-4 to Davaine's description of the bacterium causing disease in animals and man, to Koch's confirmation of this finding in 1877, and to the production of an effective attenuated-strain vaccine by Pasteur in 1881 .

The introduction of vaccination in 1965 for occupationally exposed persons and the labelling since 1978 of unsterilised bone meal have contributed to a four-fold decline in human anthrax in England and Wales in the 20-year period to 1980 . Among 145 reported cases, one was septicaemic, three were pulmonary and the others cutaneous; there were also 12 deaths. The occurrence of only 14 non-fatal cases from 1976 to 1980 showed that human anthrax is now a rare disease in Britain (Public Health Laboratory Service, 1982). The incidence in animals is also low, there being no more than a few hundred cases a year, mainly in 
cattle consuming imported feeds in the winter months.

In many countries, however, anthrax remains a major problem; it is enzootic in China, Iran, parts of Africa, India and South America, and it is virtually enzootic in many other areas. Worldwide, there may be as many as 10000 animal outbreaks each year and, perhaps, 9000 human cases. The interruption of animal vaccination, for example, in Zimbabwe by insurgency rapidly gave rise to a major bovine epizootic; a subsequent epidemic with more than 6000 human cases, most of them cutaneous, occurred and there were $c .100$ deaths in a period of only six months (Turner, 1980).

Although intestinal anthrax is rare in man, there have been several outbreaks and some of the cases reported recently in the Tropical Diseases Bulletin included an episode in which nine Ugandan children died, when 143 of 155 villagers contracted intestinal anthrax 15-72 $\mathrm{h}$ after eating a cow that had been found dead, and an outbreak that occurred in the Ivory Coast Forest seven weeks after a local epizootic in goats and sheep. In the latter incident, there were several cutaneous and two fatal cases (one intestinal and the other meningitic).

There have also been reports of oropharyngeal anthrax which is a very rare form of the disease; in a Thai outbreak that followed the consumption of infected cattle and water buffalo imported from Burma, there were 24 oropharyngeal and 52 cutaneous cases with three deaths.

Pulmonary anthrax, often referred to as "woolsorter's disease" because of its association with wool-processing plants, is usually fatal, although subclinical infections may occur. During and after the Second World War several countries showed considerable interest in B. anthracis as an air-borne agent of biological warfare, but rumours that the major epidemic in the Russian city of Sverdlovsk in 1979 was pulmonary anthrax following an explosion at a military establishment were refuted by several authorities who considered, on the basis of the scanty information available, that intestinal anthrax from contaminated meat was a more satisfactory explanation.

\section{Infections with B. cereus}

$B$. cereus, a close relative of $B$. anthracis, is undoubtedly the Bacillus species of next importance as a pathogen of man and probably of other animals also. Tracing the history of $B$. cereus infection from reports in the early literature is impracticable, however, because of problems associated with its identification and with nomenclature. Specific identification was difficult and hardly encouraged by the very confused taxonomy of the genus before the monographs of Smith and his colleagues in 1946 and 1952. Until that time and, in some instances up to the late 1960's, aerobic endospore-forming bacilli were commonly identified, if at all, as "Bacillus subtilis" or as "anthracoid rods"; the latter term may be taken to be synonymous with B. cereus. The present importance of this species and the descriptions provided in some earlier papers support the contention that many early isolates identified as $B$. subtilis were probably strains of $B$. cereus.

Recognition of more than 25 serotypes in a serotyping scheme based on flagellar antigens developed by Gilbert and his colleagues has proved useful in epidemiological studies (Gilbert, 1979; Gilbert et al., 1981).

Infections of the eye. B. cereus has emerged as one of the most virulent and destructive ocular pathogens. In 1934 François reviewed reports of 157 cases of infection of the eye by "Bacillus subtilis", although the descriptions of the organisms and their infections are suggestive rather of $B$. cereus. The six types of infection, in order of decreasing frequency, were conjunctivitis, panophthalmitis, keratitis, iridocyclitis, dacryocystitis and orbital abscess.

Panophthalmitis, usually fulminating, is the most serious of these infections; recovery of useful vision has never been reported and loss of the eye is usual. In recent years c. $50 \%$ of the cases have followed haematogenous dissemination of the organism; this has led to endogenous endophthalmitis that progressed within $24 \mathrm{~h}$ to panophthalmitis with, characteristically, severe pain, chemosis, periorbital swelling, extreme proptosis, low-grade fever, leucocytosis, ring abscess of the cornea and irreversible loss of vision. The systemic signs and ring abscess seem to be particularly associated with $B$. cereus infection. Whilst the majority of these endogenous infections occurred in intravenous drug abusers, one was associated with blood transfusion. Other cases have followed penetrating trauma, usually in environments such as farmyards and garages where soil contamination is likely. In typical cases, a metal fragment entered the eye when tools, fixtures, or pieces of machinery were being hammered; symptoms developed within $24 \mathrm{~h}$ and irreversible damage had occurred between 48 and $72 \mathrm{~h}$ (Ho et al., 1982). Some infections have followed surgical intervention. It is fortunate, therefore, that, as far as can be judged from available reports, $B$. cereus panophthalmitis is rare, relative to the frequency of penetrating injuries, intravenous drug abuse and the ubiquity of the 
organism; its incidence, however, is probably under-reported (Ho et al., 1982). In a recent and well illustrated description of a case and a review, Cowan et al. (1987) listed 15 cases that had been reported in the English literature in the past 35 years and, of these, eight followed trauma. Five of seven endogenous infections occurred in addicts and of two further, recent cases also in drug abusers, one manifested as an orbital cellulitis and the other as an acute glaucoma. Turnbull and colleagues, in analyses of exotoxin production by cultures of $B$. cereus from a wide variety of non-gastrointestinal infections, studied 13 isolates from panophthalmitis and these represented $8 \%$ of their strains (Turnbull and Kramer, 1983). Treatment has usually been unsuccessful because of the speed with which irreversible damage to the eye occurred in such a rapidly evolving infection and because of a lack of effective antibiotic therapy. If, however, early help is sought by the patient, aqueous and vitreous aspiration can be performed promptly for diagnosis and drug-sensitivity testing, and if therapy, with a combination of clindamycin and gentamicin in conjunction with cautious use of corticosteroids, is begun immediately, the patient's eye, if not his sight, may be saved (Cowan et al., 1987).

Respiratory-tract infections. Twenty-three cases of respiratory-tract infection with $B$. cereus have been reported in the last 23 years; of those cases described in detail, ten patients presented with pneumonia and four also had empyema (one was associated with infection by Clostridium bifermentans); in several cases there was an associated bacteraemia. There were isolations from three neonates with mild respiratory-tract infections and two other patients had pleurisy or a lung abscess. About $50 \%$ of the infections were fatal, especially among patients with predisposing conditions that included leukaemia, (present in five cases), alcoholism, chronic hepatitis and cirrhosis, and steroiddependent asthma. Two fatal cases with centralnervous system involvement occurred in premature babies. Heart disease may have increased the patients' susceptibilities in two cases, one of which was a mixed infection with $C$. bifermentans in an alcoholic patient and the other was a fatal infection in a normal host. Only one other infection has been reported in a normal host, who was being treated for arthritis. This too was fatal; B. cereus was isolated initially from the blood and, thereafter, from heart, lung and spleen at necropsy. B. cereus is usually resistant to penicillins and cephalosporins; pleuropulmonary infections, however, can be successfully treated with vancomycin, clindamycin and gentamicin. A report of a case and a brief review have been presented by Carbone and Stauffer (1985).

Central nervous system infections. Since 1970 there have been reports of 11 cases of meningitis caused by $B$. cereus; six of these were fatal and four were associated with bacteraemia. Six patients were infants and three who were premature died of their infections. In four cases the infection followed surgery; in two patients who had fluid shunts inserted to treat hydrocephalus, meningitis and bacteraemia resolved only after removal of the shunts; the other two patients were adults suffering from adenoma. In one, a young woman with a pituitary adenoma, treatment was successful but the other patient, who had been treated with an external shunt for rhinorrhoea, died. The latter case was described and the literature reviewed by Siegman-Igra et al. (1983). The remaining two fatal infections that occurred were in a drug abuser who was also an alcoholic, and in a young man with bacteraemia whilst undergoing chemotherapy for leukaemia.

Bacteraemia, septicaemia and endocarditis. As well as infections associated with meningitis and pneumonia (vide supra), 29 bacteraemic infections have been reported in the literature in the last 20 years and the author has information on a further four cases. B. cereus bacteraemias have been associated with a wide variety of underlying conditions but in each case there seems to have been a direct opening into a vein by which the organism probably entered the bloodstream. Several patients acquired their infections in hospital: during haemodialysis (six cases); continuous intravenous infusion following a cerebrovascular accident; hepatic perfusion in a patient with breast carcinoma who underwent cytotoxic therapy; or from other surgical procedures. Some cases of bacteraemia and septicaemia followed accidental trauma from road-traffic, industrial and domestic accidents or from burns; others were associated with drug addiction (see Borchardt et al., 1982). Of nine cases of endocarditis, six occurred in drug addicts (Siegman-Igra et al., 1983) and three were associated with heartvalve replacements (Oster and Kong, 1982; Parry et al., 1983). Despite the seriousness of these systemic infections, and the debilitated state of many of the patients, the prognoses were good and all but two, one of whom was immunocompromised by chemotherapy for leukaemia, recovered.

Wound and gangrenous infections. Wound infection by $B$. cereus is probably quite common but two problems face the clinician, and the reviewer, trying to ascertain the frequency of these cases. Firstly, it may be difficult to assess the clinical 
significance of the organism when many wound infections yield mixed cultures and, secondly, because infections with Bacillus are usually less sensational than other infections, they are less often reported in the literature. Notwithstanding, the author is aware of 111 isolations in the last 11 years from wounds and wound infections, in many of which $B$. cereus was considered significant; 22 of the infections became necrotic or gangrenous and four were fatal. There was a wide variety of wounds including multiple trauma from road-traffic accidents, crush injuries of limbs, surgical incisions, drainage sites and umbilical stumps (Turnbull et al., 1979; Barnham and White, 1980; Turnbull and Kramer, 1983). The majority of these infections were not serious but several of the gangrenous conditions required amputation (Johnson et al., 1984): severe anthracoid infections of war wounds were also noted during the Second World War (Turnbull et al., 1979). In one recent case colonisation of a minor abrasion led to fatal systemic disease in a granulocytopenic patient. It is the opinion of Barnham that moderate or heavy growths of Bacillus spp. from wounds are usually of clinical significance (Barnham and White, 1980). Although there have been several reports and retrospective studies of wound, and other, infections caused by Bacillus in which identification was not achieved or attempted, B. cereus has been by far the commonest species reported in those studies where speciation was made.

Miscellaneous infections. Other infections with $B$. cereus include two cases of osteomyelitis (Tuazon et al., 1979), one in a drug addict and the other in a patient for whom the portal of entry was unknown; both were successfully treated with, respectively, clindamycin and gentamicin.

$B$. cereus has also been isolated from infections of the female genital tract (with one case of salpingitis), from several infections of prostheses (including hip and knee joints and a heart valve), and from oto-antritis, chronic tympanitis, and abscesses in various parts of the body including the brain and lung, pustules, peritonitis and ascites. Turnbull and Kramer (1983) noted how frequently strains of serotype $\mathrm{H} 20$ were represented in their wide range of clinical isolates.

There have been three reports of $B$. cereus dissemination in maternity units with umbilical stumps being the most frequently colonised or clinically infected sites; other isolations were made from eyes, noses and, in one case, skin pustules. The majority of infections were subclinical but the potential risks were considered significant. In two outbreaks, strains of the unusual serotype H11(15) were predominant and that type and serotype $\mathrm{H} 11$ have been associated with other neonatal infections (Turnbull et al., 1979). In the third outbreak most strains belonged to serotype $\mathrm{H} 24$, a type not previously described. Although washable nappies were implicated as the primary vehicles of dissemination in one outbreak, the sources of infection in the others were uncertain (Youngs et al., 1985).

Food poisoning. Although food-poisoning outbreaks attributed to Bacillus spp. have been reported in the European literature since 1906, the first incidents in which $B$. cereus was unequivocally implicated were described by Hauge in 1950 and 1955 in his accounts of large diarrhoeal outbreaks in Norway; reproduction of the disease by feeding tests in volunteers including himself was also demonstrated then. These publications stimulated a growing awareness of the problem and the frequency of reports, most of which came from Northern and Eastern Europe, greatly increased. The illness, which is similar to that caused by $C$. perfringens, is characterised by an incubation period of 8-16 h followed by abdominal cramps, profuse watery diarrhoea and rectal tenesmus; vomiting and fever are noted occasionally. Recovery is usually complete within $24 \mathrm{~h}$ and although complications are rare, there have been two reports of associated deaths with toxic signs and lesions observed in the spleen, liver, heart, kidneys, lungs and brain at necropsy (Dirnhofer et al., 1977).

A wide variety of foods, including meat and vegetable dishes, soups, sausages, sauces and desserts, has been implicated and in many cases the dishes had been stored at room temperature for some hours before consumption. Dried foods such as milk and potato powders, starch, soup and sauce mixes and, especially, seasoning mixes and spices, are frequently heavily contaminated with the organism. In Hungary, where meat and vegetable dishes are often highly seasoned, $B$. cereus was the third most common cause of food poisoning and was responsible for $c .8 \%$ of outbreaks and $c .15 \%$ of the cases reported between 1960 and 1968 . Outbreaks have been reported from Canada, India, Japan, USA and the USSR as well as Europe but their numbers have declined in the last 15 years.

A recent major incident originating from a hospital cafeteria was reported by Baddour et al., (1986) but, as in most other outbreaks, stools were not examined for B. cereus and it is the opinion of Turnbull (1981) that the epidemiological and experimental evidence that the organism is a significant agent of diarrhoeal disease remains inadequate. Singh et al. (1984), however, described a persistent case of food poisoning in a six-month- 
old breast-fed baby; infection of the mother's breasts with a strain of B. cereus var. fluorescens, that was also repeatedly isolated from the baby's stools, was treated with gentamicin.

There were 110 incidents of $B$. cereus food poisoning reported in the United Kingdom between 1971 and 1978, and all but two of them were associated with rice, usually Chinese fried-rice dishes. Illness was characterised by the rapid onset of nausea, vomiting and malaise, and occasional diarrhoea, usually within $1-6 \mathrm{~h}$ of consumption of the contaminated food; thus, the clinical picture is similar to that found in Staphylococcus aureus food poisoning. In one case the patient developed periorbital oedema and a transient fever, but complications are rare and recovery within $24 \mathrm{~h}$ is usual. Strains of serotype $\mathrm{H} 1$ were isolated from $70 \%$ of these outbreaks; they appear to be common in cooked rice, have especially heat-resistant spores and, with strains of other serotypes often associated with the emetic syndrome but also implicated in diarrhoeal cases, form a distinct biotype (Logan et al., 1979).

In outbreaks of the emetic-type of disease the spores of $B$. cereus survive cooking of the rice, the organisms outgrow during storage, and produce toxin which is stable to, or may escape, further cooking. Thus, the syndrome is an intoxication and further cases could by prevented by the avoidance of prolonged storage of the cooked rice especially at temperatures between 15 and $50^{\circ} \mathrm{C}$. Both syndromes have been comprehensively reviewed by Gilbert et al. (1981).

\section{Toxins of $B$. cereus}

$B$. cereus produces a wide range of extracellular metabolites including exotoxins, enterotoxins, haemolysins, phospholipases, nucleases, proteases, $\beta$ lactamases, collagenase, thromboplastinase and urease; the part, if any, played by these products in the pathogenicity of the organism is unknown.

The lethality of $B$. cereus for mice has long been known and two so-called "mouse-lethal" factors have been partially characterised. One of them, a relatively unstable enterotoxigenic protein produced during vegetative growth and known variously as vascular-permeability factor, dermonecrotic toxin or fluid-accumulation factor, is thought to be involved in diarrhoeal food-poisoning. Production of this toxin appears to be correlated with the severity of non-gastrointestinal infection in man (Turnbull and Kramer, 1983) but not in bovine mastitis (Jones and Turnbull, 1981). The toxin produces increased vascular permeability and necrosis when injected intradermally in rabbits; the other "mouse-lethal" factor, a haemolysin called cereolysin, can produce similar effects and, although inactivated by serum, may also contribute to the organism's pathogenicity. When strains isolated from emetic-type food-poisoning outbreaks were grown on rice, they produced a heat-stable compound of low molecular weight that caused vomiting when fed to rhesus monkeys. Production of this emetic toxin may be peculiar to certain strains of B. cereus (vide supra). An extensive review of the toxins of $B$. cereus has been prepared (Turnbull, 1981).

\section{B. licheniformis}

B. licheniformis has been responsible for cases of ophthalmitis, a corneal ulcer following trauma that was successfully treated with gentamicin (Tabbara and Tarabay, 1979), for septicaemias and bacteraemias (one of the latter in a pregnant woman with eclampsia and acute fibrinolysis), and peritonitis with bacteraemia in an immunologically normal patient with volvulus and upper small-bowel perforation (Sugar and McCloskey, 1977). These latter infections responded to cephalosporin therapy.

Circumstantial evidence has suggested strongly that $\boldsymbol{B}$. licheniformis can cause food-poisoning manifesting as diarrhoea, sometimes with vomiting, after an incubation period of $4-15 \mathrm{~h}$. The implicated foods were mainly cooked-meat dishes in which counts of $B$. licheniformis of $10^{5}-10^{9} \mathrm{cfu} / \mathrm{g}$ of food were found (Gilbert et al., 1981; Kramer et al., 1982).

There have been many reports of the presence of L-form organisms which occur in the blood in association with erythrocytes as well as in other body fluids, and which reverted to small, acid-fast diphtheroids. On subculture or prolonged primary culture (up to 25 months) they increased in size, lost their acid-fastness and commonly reverted to grampositive endospore-forming rods, especially when grown in the presence of additives known to induce reversion of other L-forms. These organisms give licheniform colonies on agar media, are phenotypically similar to $B$. licheniformis and have been named $B$. licheniformis var. endoparasiticus (Bisset and Bartlett 1978). Several authors have claimed a positive relationship between these organisms and diseases with postulated immunological elements such as cancer and arthritis. Although some workers have reported higher isolation rates of the diphtheroids from the synovial fluids and membranes of arthritic patients than from control groups, Bartlett and Bisset (1981) obtained, in the first six months 
of culture higher isolation rates of $\boldsymbol{B}$. licheniformis var. endoparasiticus spheroplasts, diphtheroids and endospore-formers from the blood of clinically normal persons than from patients with rheumatoid arthritis; thereafter, isolation rates from both groups were similar.

\section{B. subtilis}

As already explained, early records of infection ascribed to $B$. subtilis often used that name as a synonym for "aerobic sporeforming bacilli". Since 1970 , however, there have been several reports of $B$. subtilis infection in which the identification of the species appears to have been reliable. Cases described include; endocarditis after drug abuse (cleared by cephalosporin therapy); fatal pneumonia and bacteraemia in three leukaemic patients (in two of whom the $B$. subtilis isolates were regarded as contaminants but were subsequently cultured from lung infarcts at necropsy; Pennington et al., 1976); septicaemia (cleared by gentamicin therapy) in a patient with metastasising carcinoma of the breast; infection of a necrotic axillary tumour in another breast-cancer patient; and isolations from a pleural effusion contiguous to a subphrenic abscess, from a breast prosthesis, from several surgical wound-drainage sites (Ihde and Armstrong, 1973) and from two ventriculo-atrial shunt infections, one of which was associated with bacteraemia (Tuazon et al., 1979). Furthermore, the use in laundry products of $B$. subtilis derivatives containing proteolytic enzymes has resulted in cases of dermatitis and respiratory ailments (see Norris et al., 1981).

Several episodes of food poisoning have been attributed to $B$. subtilis; it has often been found in almost pure culture in large numbers $\left(>10^{6} \mathrm{cfu} / \mathrm{g}\right)$ in the implicated foods which were usually meat dishes such as sausage rolls, meat pasties and stuffed poultry but also included pizza and wholemeal bread. Symptoms of vomiting, with diarrhoea in more than half of the incidents, occurred after incubation periods ranging from $15 \mathrm{~min}$ to $10 \mathrm{~h}$ (Gilbert et al., 1981; Kramer et al., 1982).

\section{Other Bacillus species}

B. circulans has been isolated from the cerebrospinal fluid of a neonate with fatal meningitis and from a post-operative wound infection in an aged patient with ovarian carcinoma (Logan et al., 1985). $B$. laterosporus endophthalmitis, requiring enucleation, and infections of the cornea with $B$. brevis and $B$. coagulans have been reported (Tabbara et al.,
1977); B. brevis has also been implicated in several incidents of food poisoning (Gilbert et al., 1981); and an incident of $B$. coagulans bacteraemia is on record.

Ihde and Armstrong (1973) described B. macerans wound infection after removal of a large malignant melanoma. Other reports include: cases of pustule and rectal fistula infection with $B$. pumilus (see Gilbert et al., 1981); and four isolations of B. alvei, two from cases of meningitis, one of which involved an atypical strain in a neonate (Weidermann, 1987), one from a wound infection and one associated with $C$. perfringens in gas gangrene. $B$. sphaericus has been isolated from three serious infections: a meningitis with bacteraemia and endocarditis in an alcoholic, successfully treated with penicillin; a rapidly fatal meningitis and a generalised Schwartzmann reaction in a normal subject (in neither of these cases could the portal of entry be determined); and a fatal "pseudo"-tumour of the lung in a chronic asthmatic undergoing steroid therapy (Isaacson $e t$ al., 1976).

\section{Veterinary infections}

Abortion. Association of Bacillus spp. with ovine abortion was first reported from New Zealand in 1955; ovine and bovine cases were subsequently observed in Australia in the 1960's and the ovine disease experimentally reproduced. In 1970 Ryan described two bovine cases occurring in Britain and identified the strains involved as $B$. licheniformis. Isolations of $\boldsymbol{B}$. licheniformis from ovine and bovine abortions are now regularly reported in the "Veterinary Record" by the Veterinary Investigation Service and the Scottish Veterinary Investigation Service; many of the cases occur in animals fed with silage which, especially after wet summers, is of poor quality; Atkinson (1985), however, did not find unusually high numbers of the organism in silages associated with bovine abortion. The organism has been isolated in pure culture from vaginal swabs, placentas, fetal abomasum contents, heart, liver, lung, and mediastinal and bronchial lymph nodes. Necrotising, haemorrhagic and suppurative placentitis is a common feature and the placenta has a typical brown leathery appearance; fetal pneumonia, pleurisy, pericarditis, encephalitis, hepatitis, peritonitis and thymus inflammation have also been recorded.

Pathogenesis is unclear but despite the fact that $B$. licheniformis cannot, as yet, be confirmed as the primary aetiological agent, its association with distinct placental and fetal lesions is highly suggestive of such a role (Mitchell and Barton, 1986). 
Strains from cases of abortion are phenotypically similar to those isolated from a wide variety of other sources and seem not to represent a special biotype (McGrath, Fitzsimmons and Logan, unpublished data). The author is not aware of any studies on the possibility of toxin involvement.

Cases of bovine and ovine abortion due to $B$. cereus have been reported from the USA and experimental infections by intravenous injection of pregnant ewes and heifers with the organism also resulted in abortion. Similar experiments on sheep in Australia elicited perinatal mortality and placentitis but not abortions. As with $B$. licheniformis, details of pathogenesis are unknown but necrotising and suppurative placentitis, and isolation of $\boldsymbol{B}$. cereus from placentas, fetal abomasum contents, livers, spleens, kidneys and lungs, were characteristic of these infections. Bovine, fetal suppurative pneumonia, meningoencephalitis, pericarditis, disseminated vascular emboli, lymphoreticular hyperplasia of the spleen and lymph nodes have been reported but seem to occur sporadically only (Schuh and Weinstock, 1985). The sources of the infections are unknown.

Other Bacillus species isolated from cases of abortion include: $B$. polymyxa, $B$. subtilis and unidentified species from sheep in Scotland and Norway; and B. coagulans and B. macerans from cattle in Scotland.

Mastitis. Bovine mastitis caused by $B$. cereus has been widely reported and cases have varied from mild to gangrenous with frequent rapid fatalities. Affected cows may show symptoms of depression, fever and inappetence, and death can occur within $24 \mathrm{~h}$ of the disease being noticed in peracute cases. Infected udders are swollen, hard and painful, and sometimes with purulent discharges through the wall; thereafter they become cold, darken and begin to slough as gangrene develops. The main histological findings are focal necroses of the mammary glands with numerous gram-positive bacteria in the necrotic areas; other findings have included supramammary lymphadenitis, enlarged spleen, disseminated intravascular coagulation and haemolysis with secondary haemoglobinuria. B. cereus appears to be restricted to the mammary glands and it has been suggested that lesions in other organs are due to the action of its toxins. Milk quality varies from normal to pink-tinged with blood in mild or early cases; in later, fulminating infections there may be a port-wine or rusty-brown coloured, serosanguineous discharge from which the organism is readily isolated often in pure culture. Surviving animals may be poorly productive, have fibrosis of the udder and be subject to recurrent mastitis.
In several outbreaks in Canada and the USA, intramammary administrations of contaminated antibiotic preparations were responsible for the infections. In some British cases, faeces and bedding may have been the sources after cows had been fed with contaminated brewer's grains. The ubiquity of $B$. cereus and the rarity of mastitis caused by it suggest that affected cows must in some way be predisposed or face an overwhelming challenge either from large numbers of bacteria or from especially pathogenic types. Jones and Turnbull (1981) in a report of 28 cases of bovine and one of caprine mastitis, that also reviewed the subject, did not find that their isolates produced much toxin but commented that a relatively large number of the strains were of serotype $\mathrm{H} 12$, a type not particularly associated with other sources of $B$. cereus. Treatment of $B$. cereus mastitis is often unsuccessful because of the rapidity with which the inflammation develops and the poor responses to antibiotic therapy even in some mild infections.

$B$. subtilis, $B$. licheniformis and $B$. pumilus have also been isolated in association with bovine mastitis. Fossum et al. (1986) reported 17 cases in which $B$. subtilis was considered to be the aetiological agent. Most infections were acute with general signs of ill health and pyrexia, and the surprisingly high incidences of relapse and of slaughter after failure to respond to penicillin and streptomycin therapy, to which the isolates seemed sensitive in vitro, were noted.

Other infections. Reports of other veterinary isolations of Bacillus are few but cases include: a fatal infection of a zoo tiger and two canine food poisonings by $\boldsymbol{B}$. cereus (Norris et al., 1981); and a water-borne outbreak of $B$. licheniformis infection in laboratory mice (Turnbull, 1981). Al-Mashat and Taylor (1983) found $B$. licheniformis to be common in the bovine gut and isolated strains, along with other organisms, from areas of congestion, ulceration and oedema of the mucosa; the organism was also isolated in pure culture from areas of local disruption of the mucosa that showed inflammatory cell infiltration.

"Bacillus piliformis", an endospore-forming bacterium not yet grown in vitro and which causes Tyzzer's disease in rodents, is not a recognised member of the genus; its transfer to Actinobacillus has been suggested.

\section{Identification}

Until quite recently clinical isolates of Bacillus were not identified further because they were believed to be of no clinical significance and 
because their differentiation was considered difficult and tedious. The cases mentioned in this review clearly put the wisdom of the former opinion in doubt and developments in the last decade have made identification of species of Bacillus a simple task for the routine laboratory. Bacillus species may now be characterised on the basis of a relatively small number of simple biochemical tests, or more rapidly with commercially available miniaturised test kits (API 20E and API 50CHB, API Laboratory Products, Basingstoke, Hampshire) in conjuction with morphological observations; they can also be identified by more sophisticated, but capitally

\section{REFERENCES}

Al-Mashat R R, Taylor D J 1983 Bacteria in enteric lesions of cattle. Veterinary Record 112: 5-10

Atkinson E 1985 Isolation of Bacillus licheniformis from silage associated with abortion in beef suckler herds. Journal of Applied Bacteriology 59 : xx (abstract)

Baddour L M, Gaia S M, Griffin R, Hudson R 1986 A hospital cafeteria-related food-borne outbreak due to Bacillus cereus: unique features. Infection Control 7: 462-465.

Barnham M, White D H 1980 Bacillus cereus infections. Journal of Clinical Pathology 33: 314-315.

Bartlett R, Bisset K A 1981 Isolation of Bacillus licheniformis var. endoparasiticus from the blood of rheumatoid arthritis patients and normal subjects. Journal of Medical Microbiology 14: 97-105.

Berkeley R C W, Logan N A, Shute L A Capey A G 1984 Identification of Bacillus species. In: Bergan T (ed) Methods in microbiology, Volume 16. Academic Press, London, pp 291-328.

Bisset K A, Bartlett R 1978 The isolation and characters of $L$ forms and reversions of Bacillus licheniformis var. endoparasiticus (Benedek) associated with the erythrocytes of clinically normal persons. Journal of Medical Microbiology 11: $335-349$.

Borchardt K A, Schecter G, Botch V H 1982 Bacillus cereus septicemia in a drug addict: case report. Military Medicine 147: 750-751.

Carbone J E, Stauffer J L 1985 Bacillus cereus pleuropulmonary infection in a normal host. Western Journal of Medicine 143: 676-677.

Cowan C L, Madden W M, Hatem G F, Merritt J C 1987 Endogenous Bacillus cereus panophthalmitis. Annals of Ophthalmology 19: 65-68.

Dirnhofer R, Sonnabend O, Sonnabend W 1977 Eine tödlich verlaufene lebensmittelvergiftung durch Bacillus cereus. Zeitschrift für Rechtsmedizin 80: 139-151.

Finch J E, Prince J, Hawksworth M 1978 A bacteriological survey of the domestic environment. Journal of Applied Bacteriology 45 : 357-364.

Fossum K, Kerikstad H, Binde M, Pettersen K-E 1986 Isolation of Bacillus subtilis in connection with bovine mastitis. Nordisk Veterinaermedicin 38: 233-236.

Gilbert R J 1979 Bacillus cereus gastroenteritis. In : Riemann H, Bryan F L (eds) Food borne infections and intoxications, 2nd edn. Academic Press, New York, pp 495-518.

Gilbert R J, Turnbull P C B, Parry J M, Kramer J M 1981 Bacillus cereus and other Bacillus species: their part in food expensive, methods such as pyrolysis mass spectrometry and differentiation may be achieved in all cases with the aid of a computer. The subject of identification has been comprehensively reviewed by Berkeley et al. (1984) and a colour atlas for identification of species of Bacillus is available (Parry et al., 1983). These should assist the identification of species of Bacillus isolated in significant numbers or in pure culture from specimens in which they may well prove of medical or veterinary importance. It is clear that their dismissal as of little, or no, significance is no longer an acceptable option for the aware microbiologist. poisoning and other clinical infections. In: Berkeley $\mathrm{R} \mathrm{C}$ W, Goodfellow $M$ (eds) The aerobic endospore-forming bacteria; classification and identification. Academic Press, London, pp 297-314.

Goldstein B, Abrutyn E 1985 Pseudo-outbreak of Bacillus species: related to fibreoptic bronchoscopy. Journal of Hospital Infection 6: 194-200.

Gurevitch I, Tafuro P, Krystofiak S P, Kalter R D, Cunha B A 1984 Three clusters of Bacillus pseudobacteraemia related to a radiometric blood culture analyser. Infection Control 5: $71-4$.

Ho P C, O’Day D M, Head W S 1982 Fulminating panophthalmitis due to exogenous infection with Bacillus cereus: report of 4 cases. British Journal of Ophthalmology 66: 205-208.

Ihde D C, Armstrong D 1973 Clinical spectrum of infection due to Bacillus species. American Journal of Medicine 55: 839845.

Isaacson P, Jacobs P H, Mackenzie A M R, Mathews A W 1976 Pseudotumour of the lung caused by infection with Bacillus sphaericus. Journal of Clinical Pathology 29: 806-811.

Johnson D A, Aulicino P L, Newby J G 1984 Bacillus cereusinduced myonecrosis. Journal of Trauma 24: 267-270.

Jones T O, Turnbull P C B 1981 Bovine mastitis caused by Bacillus cereus. Veterinary Record 108: 271-274.

Kramer J M, Turnbull P C B, Munshi G, Gilbert R J 1982 Identification and characterization of Bacillus cereus and other Bacillus species associated with foods and food poisoning. In: Corry $\mathrm{J}$ E $\mathbf{L}$ et al. (eds) Isolation and identification methods for food poisoning organisms. Academic Press, London, pp 261-286.

Logan N A, Capel B J, Melling J, Berkeley R C W 1979 Distinction between emetic and other strains of Bacillus cereus using the API system and numerical methods. FEMS Microbiology Letters 5: 373-375.

Logan N A, Old D C, Dick H M 1985 Isolation of Bacillus circulans from a wound infection. Journal of Clinical Pathology 38: 838-839.

Mitchell G, Barton M G 1986 Bovine abortion associated with Bacillus licheniformis. Australian Veterinary Journal 63: 160161.

Norris J R, Berkeley R C W, Logan N A, O'Donnell A G 1981 The genera Bacillus and Sporolactobacillus. In: Starr M P, et al. (eds) The prokaryotes: a handbook on habitats, isolation and identification of bacteria, Vol. 2, SpringerVerlag, Berlin and Heidelberg, pp 1711-1742.

Oster H A, Kong T Q 1982 Bacillus cereus endocarditis involving a prosthetic valve. Southern Medical Journal 75: 508-509.

Parry J M, Turnbull P C B, Gibson J R 1983 A colour atlas of 
Bacillus species. Wolfe Medical Publications, London, 272 pp.

Pennington J E, Gibbons N D, Strobeck J E, Simpson G L, Myerowitz R L 1976 Bacillus species infection in patients with hematologic neoplasia. Journal of the American Medical Association 235: 1473-1474.

Public Health Laboratory Service 1982 Anthrax surveillance 1961-80. British Medical Journal 284: 204.

Schuh J A, Weinstock D 1985 Bovine abortion caused by Bacillus cereus. Journal of the American Veterinary Medical Association 187: 1047-1048.

Siegman-Igra Y, Lavochkin J, Schwartz D, Konforti N 1983 Meningitis and bacteremia due to Bacillus cereus. Israel Journal of Medical Sciences 19: 546-551.

Singh R S, Batish V K, Parkash O M, Ranganathan B 1984 Toxigenic Bacillus cereus var. fluorescens in human milk. Journal of Dairy Science 67: 513-517.

Sugar A M, McCloskey R V 1977 Bacillus licheniformis sepsis. Journal of the American Medical Association 238: 1180-1181.

Tabbara K F, Juffali F, Matossian R M 1977 Bacillus laterosporus endophthalmitis. Archives of Ophthalmology $95: 2187-2189$.

Tabbara K F, Tarabay N 1979 Bacillus licheniformis corneal ulcer. American Journal of Ophthalmology 87: 717-719.

Tuazon C U, Hill R, Sheagren J N 1974 Microbiologic study of street heroin and infection paraphernalia. Journal of Infectious Diseases 129: 327-329.

Tuazon C U, Murray H W, Levy C, Solny M N, Curtin J A, Sheagren J N 1979 Serious infections from Bacillus sp. Journal of the American Medical Association 241 : 1137-1140.

Turnbull P C B 1981 Bacillus cereus toxins. Pharmacology and Therapeutics 13: 453-505.

Turnbull P C B, Jørgensen K, Kramer J M, Gilbert R J, Parry J M 1979 Severe clinical conditions associated with Bacillus cereus and the apparent involvement of exotoxins. Journal of Clinical Pathology 32: 289-293.

Turnbull P C B, Kramer J M 1983 Non-gastrointestinal Bacillus cereus infections: an analysis of exotoxin production by strains isolated over a two year period. Journal of Clinical Pathology 36: 1091-1096.

Turner M 1980 Anthrax in humans in Zimbabwe. Central African Journal of Medicine 26: 160-161.

Twigg G 1984 The black death: a biological reappraisal. Batsford, London, 254pp.

Weidermann B L 1987 Non-anthrax Bacillus infections in children. Pediatric Infectious Disease Journal 6: 218-219.

Youngs E R, Roberts C, Kramer J M, Gilbert R J 1985 Dissemination of Bacillus cereus in a maternity unit. Journal of Infection 10: 228-232. 